\title{
Acoustic oscillations and dynamo action in the G8 sub-giant EK Eridani ${ }^{\star}, \star \star$
}

\author{
A. Bonanno ${ }^{1}$, E. Corsaro ${ }^{1}$, F. Del Sordo ${ }^{2,3}$, P. L. Pallé ${ }^{4}$, D. Stello ${ }^{5}$, and M. Hon ${ }^{5}$ \\ ${ }^{1}$ INAF - Osservatorio Astrofisico di Catania, Via S. Sofia, 78, 95123 Catania, Italy \\ e-mail: enrico.corsaro@inaf.it, alfio.bonanno@inaf.it \\ 2 Institute of Astrophysics, FORTH, 71110 Heraklion, Greece \\ 3 Department of Physics, University of Crete, 70013 Heraklion, Greece \\ ${ }^{4}$ Instituto de Astrofísica de Canarias, 38205 La Laguna, Tenerife, Spain \\ 5 School of Physics, University of New South Wales, Sydney, NSW 2052, Australia
}

Received 3 May 2019 / Accepted 2 July 2019

\begin{abstract}
We present further evidence of acoustic oscillations in the slowly rotating overactive G8 sub-giant EK Eri. This star was observed with the $1 \mathrm{~m}$ Hertzsprung SONG telescope at the Observatorio del Teide for two different runs of 8 and 13 nights, respectively, that were separated by about a year. We determined a significant excess of power around $\nu_{\max }=253 \pm 3 \mu \mathrm{Hz}$ in the first observing run and were able to determine a large separation, $\Delta v=16.43 \pm 0.22 \mu \mathrm{Hz}$. No significant excess of power was instead detected in a subsequent SONG observing season, as also supported by our analysis of the simultaneous TESS photometric observations. We propose a new amplitude-luminosity relation in order to account for the missing power in the power spectrum. Based on the evolutionary stage of this object, we argue that a standard $\alpha^{2} \Omega$ dynamo cannot be excluded as the possible origin for the observed magnetic field.
\end{abstract}

Key words. stars: activity - starspots - stars: rotation - asteroseismology - magnetohydrodynamics (MHD) stars: individual: EK Eri

\section{Introduction}

Although with different physical emphasis, both magnetic fields and acoustic oscillations are ubiquitous along the HertzsprungRussell diagram. In the case of the Sun, the activity level is correlated with the frequencies and power of the $p$-mode oscillations (Woodard \& Noyes 1985; Palle et al. 1989; Libbrecht \& Woodard 1990; Elsworth et al. 1990). In recent times, it has been possible based on the high-quality data of the CoRoT and Kepler missions to investigate the effect of magnetic activity in solar-like stars in a systematic fashion (e.g., see García et al. 2010). Chaplin et al. (2011) analyzed a sample of about 2000 solar-like stars, and evidence of pulsations was found in only about 540 stars. In particular, it was found that as the activity level increased, the number of detections decreased. A further analysis that made use of the Mount Wilson S-index chromospheric activity indicator reported an anticorrelation between the S-index and the amplitude of the acoustic modes (Bonanno et al. 2014). A possible explanation is the decrease of turbulent velocities near the surface as a result of the magnetic field that reduces the efficiency of the convection (Dziembowski \& Goode 2005; Jacoutot et al. 2008).

* A table of the radial velocities is only available at the CDS via anonymous ftp to cdsarc.u-strasbg.fr $(130.79 .128 .5)$ or via http://cdsarc.u-strasbg.fr/viz-bin/qcat?J/A+A/628/ A106

$\star \star$ Based on observations made with the Hertzsprung SONG telescope operated on the Spanish Observatorio del Teide on the island of Tenerife by the Aarhus and Copenhagen Universities and by the Instituto de Astrofísica de Canarias.
The relevant question is understanding the physical mechanism that underlies this process. The G8 sub-giant EK Eri (HR 1362, HD 27536) represents a unique opportunity to do this (we note that although the star is a spectroscopically determined sub-giant, its characteristic oscillation frequency, $v_{\max }$, places it firmly on the red giant branch). As first noted by Dall et al. (2010), the analysis of high-precision radial velocities obtained with the HARPS spectrograph has revealed a significant suppression of the oscillation modes.

Because of its slow rotation $\left(v \sin i<1 \mathrm{~km} \mathrm{~s}^{-1}\right)$, brightness variations are consistent with a rotational period of $\sim 308$ days because star spots are rotated across the projected surface of the star (Strassmeier et al. 1990, 1999). Spectropolarimetric observations (Aurière et al. 2008, 2011) have determined that the magnetic field geometry is dominated by a large-scale almost completely dipolar field with a strength of $\sim 200-250 \mathrm{G}$. This object is therefore significantly overactive with respect to its rotation rate and evolutionary state. It is strongly different from the normal period-activity relation (Aurière et al. 2015).

The physical explanation for the observed field in this object is still much debated. When conservation of the magnetic flux is assumed, EK Eri on the zero-age main sequence (ZAMS) would have had a field strength of a few kilogauss, which is well within the typical range for a magnetic Ap star (Stepien 1993). However, this simple assumption is almost certainly inadequate because as the star evolves off the main sequence, the growing outer convection zone interacts with the original field. Alternatively, a fossil field that acted as the seed for a growing dynamo-generated field may have emerged. The abundance pattern determined by Dall et al. (2010) is very similar to the solar abundance pattern, however, which suggests that none of the anomalies could be 
attributed to the previous evolutionary status as a magnetic Ap star.

Alternatively, in spite of its very low equatorial velocity, EK Eri might have produced enough differential rotation to host a genuine dynamo that generated the observed field. A recent investigation with Kepler data has shown that in contrast to the well-established behavior at small Rossby numbers (the ratio of rotation period to the convective turnover time), the chromospheric activity of the more slowly rotating stars of the open cluster M 67 has been found to increase with increasing Rossby number (Brandenburg \& Giampapa 2018). According to Brandenburg \& Giampapa (2018), slow rotators with high stellar activity might indeed be characterized by antisolar differential rotation.

The study of the acoustic spectrum of EK Eri will provide essential information to settle the question. Asteroseismology can precisely determine the evolutionary status of the star and therefore constrain its internal density stratification and determine the strength of the core-envelope coupling from the spectrum of mixed modes. However, the severe violation of the scaling relation for the amplitude (Kjeldsen \& Bedding 1995) when compared to the value measured by Dall et al. (2010), if properly interpreted, opens up a window into the details of the excitation mechanism of the acoustic modes in the presence of a strong surface magnetic field.

The new radial velocity measurements made with the Stellar Observations Network Group (SONG) spectrograph that were obtained in two different observational campaigns will provide further evidence for the presence of $p$ modes in this star. Although it is strongly suppressed, the location of the excess of power in the power spectrum allowed us to obtain an estimate for the large separation in this star.

We therefore used this information to develop an internal model of the star, which we used in a spherical dynamo model to study possible dynamo actions in this object. We show that an $\alpha^{2} \Omega$ dynamo can be operative even if the Rossby number near the base of the convection zone is greater than one. The resulting magnetic field topology is close to the observed topology.

We further suggests a modification of the standard amplitude-scaling relation of Kjeldsen \& Bedding (1995) that takes into account the missing convective flux that is due the presence of a large spotted area. We argue that the nondetection of the oscillation modes in the second observational campaign is a consequence of a further decrease of the convection efficiency that is due to the strong fluctuations of the magnetic field, as observed in Aurière et al. (2011).

The structure of the paper is as follows: in Sect. 2 we describe the observations and data reduction, Sect. 3 describes the results, Sect. 4 contains the results of a kinematic dynamo model applied to the star, and Sect. 5 is devoted to the conclusions.

\section{Observations}

Dall et al. (2010) reported the first detection of solar-like oscillations in EK Eri based on about three nights of high-precision radial velocity observations with HARPS. However, the short length of their observation set only allowed them to estimate the frequency of the maximum oscillation power, $v_{\max }$, which they found to be $320 \pm 32 \mu \mathrm{Hz}$. Following this detection, we decided to perform new observations of the target to be able to better characterize its $v_{\max }$ and if possible, derive its large frequency separation $\Delta v$ for the first time.

The new radial velocity time-series obtained for EK Eri come from observations made with the $1 \mathrm{~m}$ Hertzsprung SONG telescope at the Observatorio del Teide, for which its ećhelle spectrograph was used. The Hertzsprung telescope constitutes the first node of SONG. Observations were conducted for a total of about eight consecutive nights starting from 2 December 2017 with $\sim 312$ individual data points, which means that we had about 40 points per night (see Fig. 1). The spectra were reduced and calibrated using the SONG pipeline and an iodine cell for precise wavelength calibration. For more details about the characteristics and reduction pipeline of the Hertzsprung telescope, we refer to Andersen et al. (2014) and Grundahl et al. (2017). Because the star has an apparent magnitude of $V \simeq 6.1$, we chose a spectral resolution of 90000 and an exposure time of 600 s throughout the run. A second observing run of $\sim 13$ nearly consecutive nights was also performed in 2018, and we discuss its results in Sect. 3.3.

\subsection{Time-series analysis}

The power spectrum of the velocity time-series was calculated as both an unweighted and weighted least-squares fit of sinusoidal components following Corsaro et al. (2012; see also Frandsen et al. 1995; Arentoft et al. 1998; Bedding et al. 2004; Kjeldsen et al. 2005; Creevey et al. 2019). The resulting power was then converted into the power spectral density (PSD) by normalizing for the spectral resolution, which is calculated as the integral of the spectral window of the observing set, here corresponding to $\sim 4.87 \mu \mathrm{Hz}$. The purpose of testing the case of a weighted least-squares spectrum is to determine any improvements in the signal-to-noise ratio $(\mathrm{S} / \mathrm{N})$, which is relevant in order to increase the chances of detecting an oscillation envelope. Similarly to what has been done previously in Corsaro et al. (2012), we assigned a weight to each point of the radial velocity timeseries that was given by the corresponding uncertainty estimate from the SONG pipeline. Because radial velocity uncertainties provided by the SONG pipeline might not necessarily be realistic, we have corrected them for possible outliers following the approach presented by Butler et al. (2004) that was also adopted by Corsaro et al. (2012). By measuring the amplitude of the noise level in the amplitude spectrum in the region $300 \mu \mathrm{Hz}$ up to the Nyquist frequency of $\sim 822 \mu \mathrm{Hz}$, we found that the amplitude of the noise is $34.0 \mathrm{~cm} \mathrm{~s}^{-1}$ and the maximum amplitude around $v_{\max }$ is $99.8 \mathrm{~cm} \mathrm{~s}^{-1}$ in the unweighted case, while we have $33.5 \mathrm{~cm} \mathrm{~s}^{-1}$ and $106.0 \mathrm{~cm} \mathrm{~s}^{-1}$, respectively, in the weighted case. The signalto-noise ratio is slightly improved in the weighted spectrum, therefore we adopted this spectrum for the subsequent asteroseismic analysis.

\subsection{Extraction of global seismic parameters}

Similarly to Creevey et al. (2019), we used the DiAmonds code (Corsaro \& De Ridder 2014) for the Bayesian inference of the PSD of EK Eri. The model adopted consists of a simple flatnoise component and a Gaussian envelope to model the oscillation power excess (Corsaro \& De Ridder 2014). To identify the power excess, we searched in a region close to the first $v_{\max }$ estimate published by Dall et al. (2010). This region can also be identified by means of the spectroscopic properties of $\log g$ and $T_{\text {eff }}$ obtained by Dall et al. (2010) because $v_{\max } \propto$ $\mathrm{g} / \sqrt{T_{\text {eff }}}$ (Brown et al. 2011). With the new estimate for $v_{\max , \odot}$ by Fredslund Andersen et al. (2019) to scale from the Sun, the spectroscopic values yield $v_{\max } \approx 300 \mu \mathrm{Hz}$. This is well below the limiting Nyquist frequency of our dataset. The resulting fit with Diamonds is shown in Fig. 2 (top panel), where the Gaussianshape hump of the oscillations is centered at $v_{\max }=253 \pm 3 \mu \mathrm{Hz}$, 


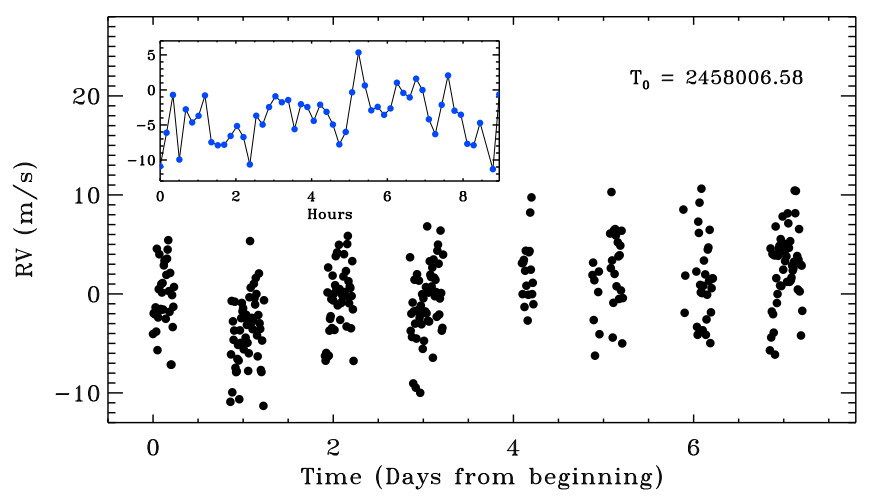

Fig. 1. Radial velocity time-series of EK Eri with SONG for the entire run in 2017; the details of the second observing night are shown in the inset. The time $T_{0}$ (BJD) of the first data point of the entire campaign is also indicated.

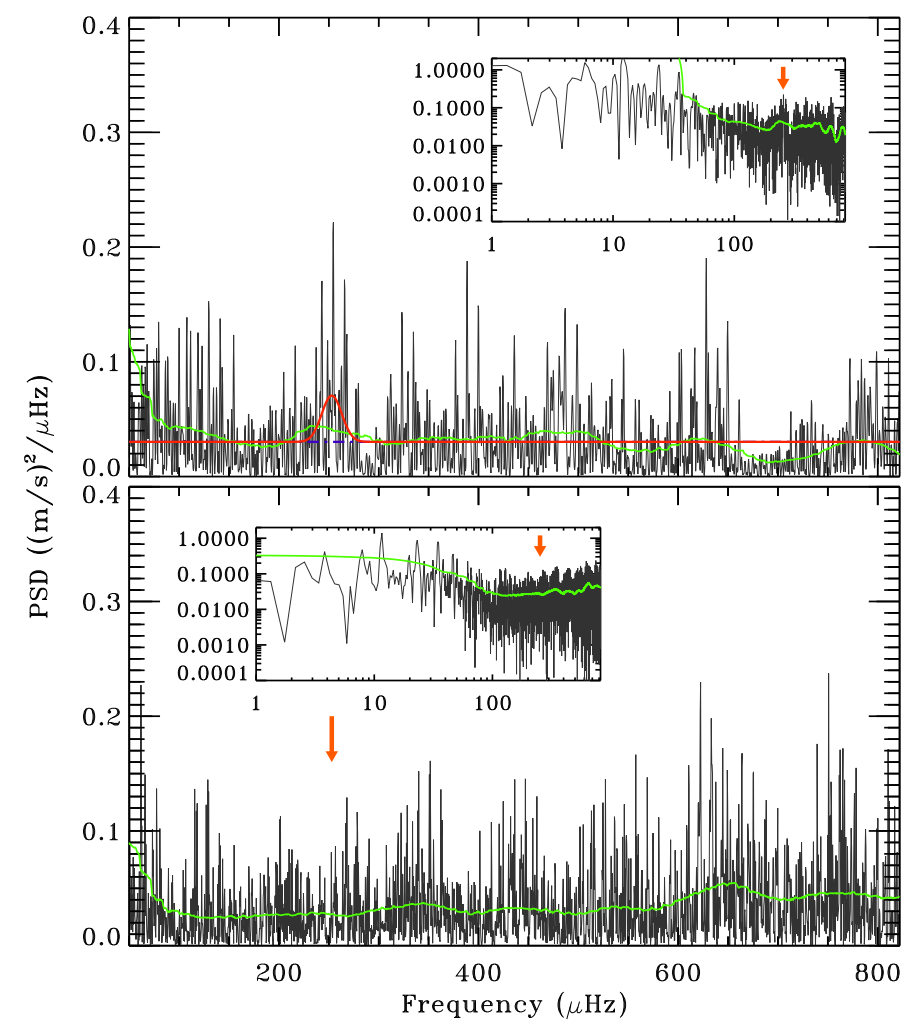

Fig. 2. Top panel: power spectral density (in black) and background model fit using DiAmonds for EK Eri from the eight-night observation run in 2017. The red line shows the total fit including the oscillation power excess, and the dashed blue line below the oscillation hump represents the flat-noise component. The inset shows the same spectrum, but in a log-log scale to help visualize the hump that is caused by oscillations, which is also marked by a red arrow at $v_{\max }$. A smoothing by $4 \Delta v$, with $\Delta v$ derived from Stello et al. (2009) using $v_{\max }$ from our analysis (solid green line), is also shown. Bottom panel: similar to the top panel, but here using the second observation run in 2018. The position of $v_{\max }$ from the 2017 time-series is indicated by the red arrows, showing that there is no region where significant power excess is found.

with the uncertainty corresponding to the $68.3 \%$ Bayesian credible interval (see Table 1 for a list of all the properties of this star). We additionally tested the significance of this region by means of a statistical model comparison against (1) a nondetection case (only flat noise); and (2) the selection of a different power hump, one at $330 \mu \mathrm{Hz}$ and another around $130 \mu \mathrm{Hz}$, which represent the

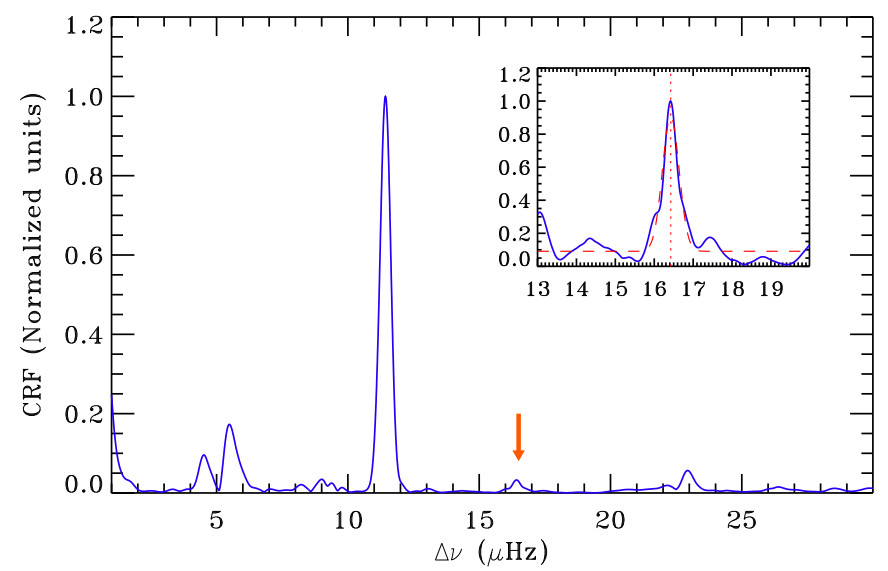

Fig. 3. CRF of the region of the PSD of EK Eri centered around $v_{\max }$ (in blue) for a search range between 1 and $30 \mu \mathrm{Hz}$, where multiples of the daily aliasing at $11.57 \mu \mathrm{Hz}$ are clearly visible. The arrow indicates the peak corresponding to the position of the actual $\Delta v$. The inset shows the result of applying the CRF to a smaller frequency range that excludes the main peaks from the daily aliases, between 13 and $20 \mu \mathrm{Hz}$. In the inset, the red dashed curve represents a Gaussian fit to the CRF peak; the centroid and standard deviation of the Gaussian mark the value of $\Delta v$ and its corresponding uncertainty, as used in this analysis.

two closest neighbors to the one originally selected. In all cases we investigated, the result based on comparing the Bayesian evidence of each model (with and without the Gaussian envelope and between the different power humps we selected) yields a strong evidence condition ( $\ln B>5$, where $B$ is the Bayes factor) in favor of the detection of the oscillation envelope centered at $v_{\max }=253 \mu \mathrm{Hz}$.

In addition to a statistical validation of the power excess, we estimated the characteristic frequency of the granulation component, $b_{\text {gran }}$, using the photometric Transiting Exoplanet Survey Satellite (TESS) observations of EK Eri (see Sect. 3.4 for more details). This approach provides an entirely independent verification of the expected value of $v_{\max }$ for our star because $b_{\text {gran }}$ and $v_{\max }$ are tightly related to one another (see, e.g., Kallinger et al. 2014). By performing a background fit with Diamonds to the TESS PSD (Corsaro et al. 2017), we have that $b_{\text {gran }}=230 \mu \mathrm{Hz}$. By inverting the power law that links $b_{\text {gran }}$ to $v_{\max }$, as obtained by Kallinger et al. (2014), we have $v_{\text {max,pred }}=253 \mu \mathrm{Hz}$, which agrees excellently with our estimate from the SONG spectrum.

To determine the value of $\Delta v$, we instead focused on the region of the oscillations and computed a comb response function (CRF) out of a set of the ten highest signal-to-noise ratio frequencies extracted by means of a CLEAN algorithm in the PSD region $200-300 \mu \mathrm{Hz}$. For this purpose, we adopted a similar procedure as was presented in Bonanno et al. (2008) that was later also applied by Corsaro et al. (2012). We first selected a wide range of values for $\Delta v(1-30 \mu \mathrm{Hz})$ in order to clearly locate a possible peak that is different than the multiples of the daily aliasing, $11.57 \mu \mathrm{Hz}$. Then, we restricted our search range to a region where $\Delta v$ from scaling relations is expected (e.g., see Stello et al. 2009), hence excluding the daily aliases at 11.57 and twice this value in order to enhance the signal of the expected $\Delta v$ peak. The result is presented in Fig. 3, which shows a peak of the CRF at $\Delta v=16.43 \pm 0.22 \mu \mathrm{Hz}$.

Last, we also determined the maximum amplitude of the radial oscillations, $A_{\mathrm{osc}}$, following the recipe presented by Kjeldsen et al. (2005; but see also Corsaro et al. 2012). The 
uncertainty reported in Table 1 is here obtained by incorporating the uncertainty on the background level of the PSD.

\section{Results}

\subsection{Derivation of fundamental stellar properties}

Because a Gaia DR2 parallax is available (Lindegren et al. 2018), we first computed a stellar radius based on this information, exploiting the interstellar reddening from the Panoramic Survey Telescope and Rapid Response Survey (PanSTARRS-1) 3D galaxy map (Green et al. 2015), the extinction-to-reddening ratio for stars with high galactic latitude from Fitzpatrick (1999), and a bolometric correction from Flower (1996) for a $T_{\text {eff }}=$ $5135 \mathrm{~K}$ star (Dall et al. 2010). The result obtained is $R_{\mathrm{GDR} 2}=$ $4.91 \pm 0.13 R_{\odot}$. We note that although the Gaia DR2 parallax is not within $1 \sigma$ of the Hipparcos parallax for the same star, we still find a good agreement (well within $1 \sigma$ ) between our estimate of the stellar radius based on the Gaia DR2 parallax and that obtained by Dall et al. (2010) using the Hipparcos parallax.

After we obtained the global seismic parameters, we derived an estimate of the stellar mass and radius from asteroseismic scaling relations (e.g., Kjeldsen \& Bedding 1995) using the relations

$$
\begin{aligned}
M / M_{\odot} & =\left(\frac{v_{\text {max }}}{v_{\text {max }, \odot}}\right)^{3}\left(\frac{\Delta v}{\Delta v_{\odot}}\right)^{-4}\left(\frac{T_{\text {eff }}}{T_{\text {eff } \odot}}\right)^{1.5} \\
R / R_{\odot} & =\left(\frac{v_{\text {max }}}{v_{\text {max }, \odot}}\right)\left(\frac{\Delta v}{\Delta v_{\odot}}\right)^{-2}\left(\frac{T_{\mathrm{eff}}}{T_{\text {eff }, \odot}}\right)^{0.5}
\end{aligned}
$$

for mass and radius, respectively, where we adopted $T_{\text {eff, } \odot}=$ $5777 \mathrm{~K}$ and the new asteroseismic solar-SONG reference values (Fredslund Andersen et al. 2019), that is, $\nu_{\max , \odot}=3141 \pm 12 \mu \mathrm{Hz}$, $\Delta v_{\odot}=134.98 \pm 0.04 \mu \mathrm{Hz}$. We note that the seismic radius obtained in this work, $R_{\text {seismic }}=5.12 \pm 0.15 R_{\odot}$, is compatible (within $1 \sigma$ ) with that derived using the Gaia DR2 parallax, and it has a precision of about $3 \%$. We derive a seismic mass of $M_{\text {seismic }}=2.00 \pm 0.14 M_{\odot}$, which with a precision of about $7 \%$ is in agreement with previous estimates proposed by Aurière et al. (2008), and in particular with the measurement obtained by Dall et al. (2010) (well within $1 \sigma$ ). We also note that this value agrees with a mass derived using the spectroscopic $\log g$ from Dall et al. (2010) and the new stellar radius obtained from Gaia DR2, corresponding to about $2.16 M_{\odot}$. However, we point out that standard (uncorrected) seismic scaling relations have the tendency to overestimate stellar radius and mass when applied to evolved stars (see, e.g., Gaulme et al. 2016; Sharma et al. 2016). To mitigate this problem, Kallinger et al. (2018) recently developed new nonlinear scaling relations. In order to determine possible inconsistencies, we adopted these nonlinear relations for EK Eri and obtained $R_{\text {non-linear }}=4.96 \pm 0.14 R_{\odot}, M_{\text {non-linear }}=1.84 \pm 0.12 R_{\odot}$. These values, although systematically lower, appear to still agree (within $1 \sigma$ ) with radius and mass estimates obtained from the standard scaling relations adopted in this work. The nonlinear scaling estimates are also compatible (within $1 \sigma$ ) with literature values from Aurière et al. (2008), Dall et al. (2010) and with the Gaia DR2 radius and $\log g$-based mass. By exploiting the new Gaia parallax to derive the stellar luminosity, we find that the stellar luminosity agrees with the value derived by Dall et al. (2010), but with the advantage that we estimated it with a precision of about $2.3 \%$, as opposed to the precision of about $11 \%$ of the previous value.
Table 1. Observed properties of EK Eri.

\begin{tabular}{llll}
\hline \hline Property & Units & Value & Source \\
\hline$v \sin i$ & $\mathrm{~km} \mathrm{~s}^{-1}$ & $<1.6 \pm 0.4$ & Dall et al. (2010) \\
$P_{\text {rot }}$ & $\mathrm{d}$ & $308.8 \pm 2.5$ & Dall et al. (2010) \\
$T_{\text {eff }}$ & $\mathrm{K}$ & $5135 \pm 60$ & Dall et al. (2010) \\
$\log g$ & & $3.39 \pm 0.12$ & Dall et al. (2010) \\
{$[\mathrm{Fe} / \mathrm{H}]$} & $\mathrm{dex}$ & $+0.02 \pm 0.04$ & Dall et al. (2010) \\
\hline$E(B-V)$ & & 0.006 & Green et al. (2015) \\
$A_{v} / E(B-V)$ & & 3.1 & Fitzpatrick (1999) \\
$B C$ & & -0.25 & Flower (1996) \\
$\pi_{\mathrm{GDR} 2}$ & $\mathrm{mas}$ & $15.58 \pm 0.05$ & Lindegren et al. (2018) \\
$R_{\mathrm{GDR} 2}$ & $R_{\odot}$ & $4.91 \pm 0.13$ & This work \\
$L$ & $L_{\odot}$ & $15.07 \pm 0.35$ & This work \\
\hline$v_{\text {max }}$ & $\mu \mathrm{Hz}$ & $253 \pm 3$ & This work \\
$\Delta v$ & $\mu \mathrm{Hz}$ & $16.43 \pm 0.22$ & This work \\
$A_{\text {osc }}$ & $\mathrm{m} \mathrm{s}$ & $0.22 \pm 0.01$ & This work \\
$R_{\text {seismic }}$ & $R_{\odot}$ & $5.12 \pm 0.15$ & This work \\
$M_{\text {seismic }}$ & $M_{\odot}$ & $2.00 \pm 0.14$ & This work \\
\hline
\end{tabular}

\subsection{Suppression of the oscillation amplitude}

As shown for instance by García et al. (2010) and Chaplin et al. (2011), surface magnetic activity has the effect of suppressing the amplitude of solar-like oscillations because the convection mechanism becomes less efficient. By considering the scaling relation for oscillation amplitudes in radial velocity, proposed originally by Kjeldsen \& Bedding (1995),

$A_{\mathrm{osc}}=\frac{L / L_{\odot}}{M / M_{\odot}}(23.4 \pm 1.4) \mathrm{cm} \mathrm{s}^{-1}$,

and based on our derivation of the stellar luminosity and mass, we evaluate that the expected oscillation amplitude for EK Eri would be $A_{\mathrm{osc}}=1.79 \pm 0.11 \mathrm{~m} \mathrm{~s}^{-1}$. This value is clearly significantly higher than what we measured from the actual PSD. It is higher by a factor of about eight, while it appears close to the value measured by Dall et al. (2010), although it is about $30 \%$ higher.

We can interpret this fact in the following way. If the star has a large spotted area, the effective luminosity responsible for the amplitude in Eq. (3) is not $L$, but $L-S \sigma T^{4}$, where $S$ is the area of the spot and $\sigma$ is the Stefan-Boltzmann constant. Therefore Eq. (3) can be rewritten as

$A_{\mathrm{osc}}^{\mathrm{spotted}}=\beta \frac{L / L_{\odot}}{M / M_{\odot}}(23.4 \pm 1.4) \mathrm{cm} \mathrm{s}^{-1}$,

where

$\beta=\frac{L-\Delta L}{L}=1-\frac{S}{4 \pi R^{2}}$

because $\Delta L=S \sigma T^{4}$. When we use our information about the suppression factor of the $p$-mode amplitude, we can easily invert Eq. (4) and estimate the effective spotted area, which is $88 \pm 6 \%$ of the total surface of the star. It is encouraging to note that this estimate agrees roughly with the findings of Aurière et al. (2011; see in particular the topology of the radial field in their Fig. 6). Clearly, for a much less active star like the Sun, $\beta \approx 1$, and we recover the standard scaling relation.

We note that when a Kepler calibrated amplitude-scaling relation such as the one proposed by Corsaro et al. (2013) (converted into a radial velocity amplitude according to the 


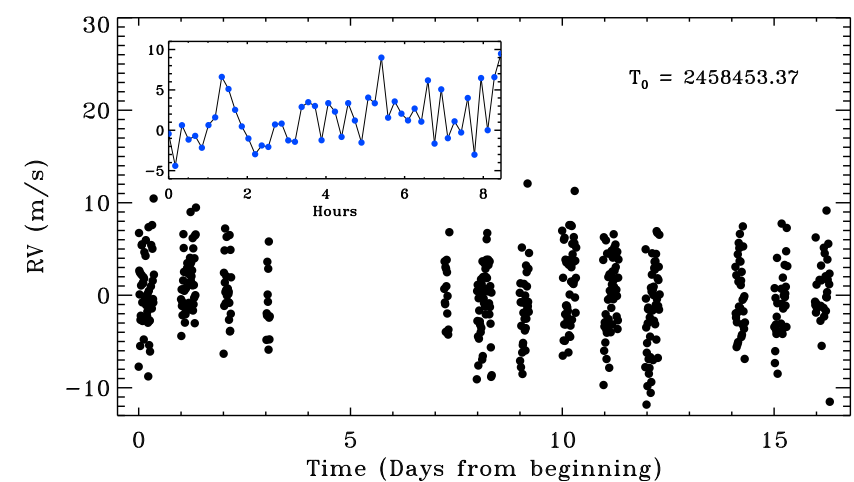

Fig. 4. Radial velocity time-series of EK Eri with SONG for the entire run in 2018; the detail of the second observation night is shown in the inset. The time $T_{0}$ (BJD) of the first data point of the entire campaign is also indicated. No clear periodicity in the signal is visible.

relation by Kjeldsen \& Bedding 1995) is used, we obtain $A_{\text {osc }}=1.24 \mathrm{~m} \mathrm{~s}^{-1}$, which is $30 \%$ lower than that predicted using Eq. (3). Relative to this expected oscillation amplitude, the observed amplitude suppression is still a factor of almost six, and the spotted area estimated through Eq. (5) remains about $80 \%$ of the total visible surface of the star. However, we recall that the conversion from bolometric into radial velocity amplitude following Kjeldsen \& Bedding (1995) is still not fully understood and that the derived $A_{\mathrm{osc}}$ in this latter case might be overestimated (see, e.g., Huber et al. 2011a).

\subsection{Missing oscillatory signal in the second SONG time-series}

In addition to the first observations of EK Eri that we presented in Sect. 2, we attempted to observe the star one year later, using the same SONG instrumental setup, in order to gather a longer time-series and thus obtain a better frequency resolution in the resulting PSD. The new observations were conducted between 30 November 2018 and 17 December 2018, covering a total of about 13 nights and nearly 480 individual radial velocity measurements (see Fig. 4 for more details on the distribution of individual observation nights).

Observing conditions were very similar to the first observations in 2017, and so was the noise level measured in the data, which is found to be $31.5 \mathrm{~cm} \mathrm{~s}^{-1}$ as measured in the region from $300 \mu \mathrm{Hz}$ up to the Nyquist frequency. Nonetheless, by processing the time-series in the same manner as presented in Sect. 2.1, we did not find any evidence for a power excess due to the oscillations in the frequency region of $v_{\max }$ from Table 1 , as is visible in the new PSD of the star (see Fig. 2, bottom panel). Because the noise level of the new dataset is not higher than in 2017, we can exclude the noise in the PSD as the possible cause of a nondetection. In this regard, Aurière et al. (2011) noted that the magnetic field is subject to fluctuations on the order of $80 \mathrm{G}$ from one year to another (see also their Table 1). We deem these fluctuations the likely cause of a variation in the level of suppression of the oscillations and therefore of the missing oscillation power excess. According to the previous discussion, even a small increase in $\beta$ can render the amplitude of the modes not observable because the level of the $\mathrm{S} / \mathrm{N}$ is already low.

\subsection{New TESS observations}

From 15 November until 11 December 2018, EK Eri (TIC 37778297) was monitored by the recently launched NASA

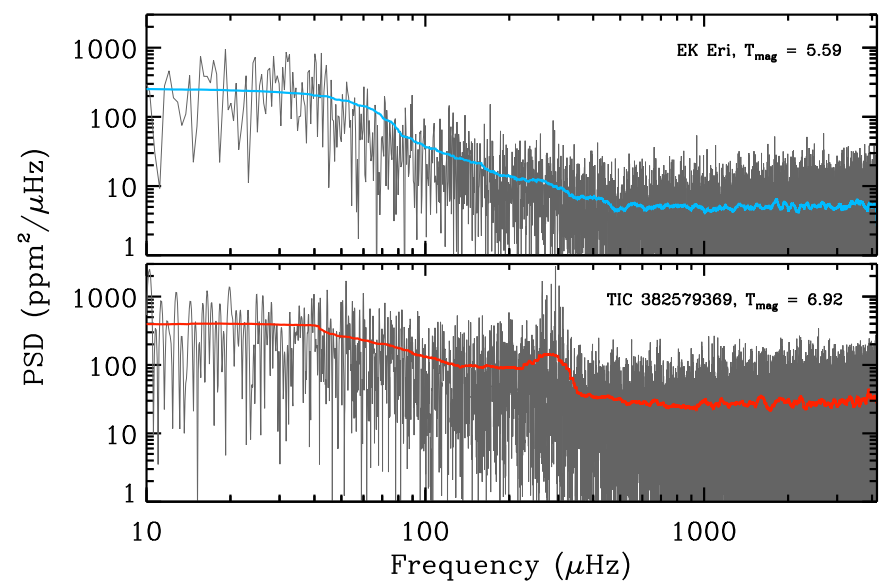

Fig. 5. Upper panel: PSD (in gray) for EK Eri using the TESS observations from sector 5. Lower panel: PSD of TIC 382579369, a star with a similar magnitude and $v_{\max }$ to that of EK Eri. The colored solid lines represent the smoothing by $4 \Delta v$, similarly to Fig. 2 .

mission TESS (Ricker et al. 2014) in observing sector 5. This photometric observation is essentially simultaneous to that of our second SONG time-series that we presented in Sect. 3.3. We can therefore assess the detectability of the oscillation envelope from both photometric and radial velocity datasets. To further aid this assessment, we also analyzed the TESS data of a star with similar $v_{\max }$ and brightness as EK Eri. This serves as a comparison star. We applied $4 \sigma$ clipping to the TESS light curves and passed them through a high-pass filter ( $9.3 \mathrm{~h}$-wide boxcar) with a cutoff frequency of $30 \mu \mathrm{Hz}$ to remove long-term trends. Finally, the light curves were gap-filled using the in-painting algorithm (Pires et al. 2015) to reduce the effect of spectral leakage of high power at low frequencies that could otherwise obscure the power-frequency profile of solar-like oscillations. The resulting power spectrum of EK Eri as observed with TESS is shown in Fig. 5 (upper panel), where we also plot the TESS comparison star, TIC 382579369 (lower panel). The comparison between the two shows that the lack of a clear oscillation power excess in EK Eri is not the result of the star being too faint (the comparison star is even slightly fainter), but rather that EK Eri shows strongly suppressed oscillations. The absence of an oscillation bump in the TESS power spectrum of EK Eri therefore supports the conclusions drawn from the analysis of our second SONG time-series.

To further quantify the level of suppression, we also performed a background fit to the power density spectrum of TIC 382579369 with Diamonds, using the same approach as adopted by Corsaro et al. $(2015,2017)$. In this way, we estimated the photometric amplitude of our TESS comparison star following the same procedure as used by Kjeldsen \& Bedding (1995). We show the result in Fig. 6, where the amplitude of TIC 382579369, of $22 \mathrm{ppm}$ (star symbol), is compared to a sample of Kepler stars ranging from the main sequence (high $v_{\max }$ ) to near the tip of the red giant branch (low $v_{\max }$ ) that were previously analyzed by Huber et al. (2011b) using the same method (see also Corsaro et al. 2013). In the same plot, we also show our estimated bolometric amplitude for EK Eri, $4.38 \mathrm{ppm}$, as obtained by converting the radial velocity amplitude from the SONG 2017 observing campaign into a bolometric amplitude according to Kjeldsen \& Bedding (1995). It is clear that while the amplitude of TIC 382579369 is in line with the "normal" trend from the Kepler sample, EK Eri falls significantly below what is expected for a star with a $v_{\max }$ of about $200-300 \mu \mathrm{Hz}$. 


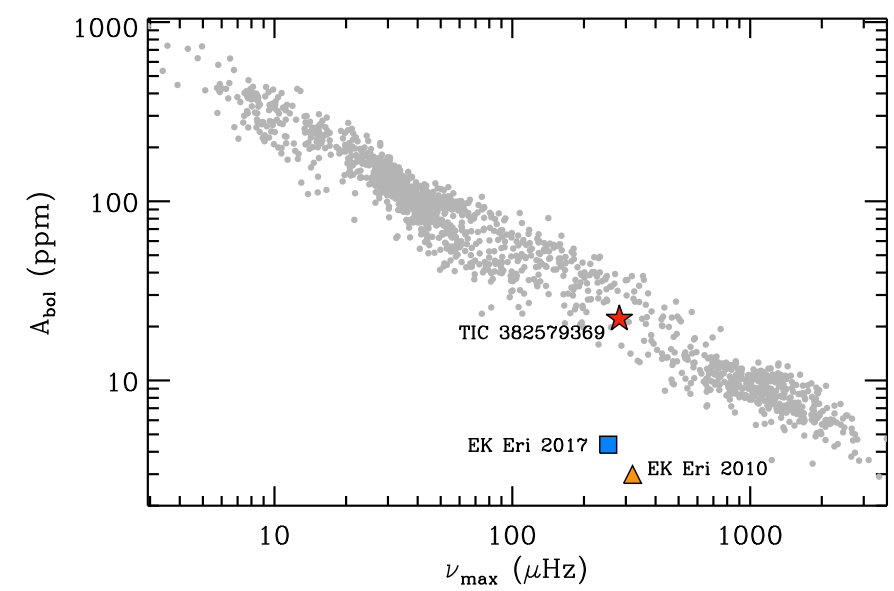

Fig. 6. Bolometric oscillation amplitudes for a sample of 1640 Kepler stars (gray dots) analyzed by Huber et al. (2011b). The red star marks the amplitude of the comparison star TIC 382579369 (Fig. 5, lower panel) as measured in this work, while the blue square shows the amplitude of EK Eri obtained by converting its radial velocity amplitude into a bolometric amplitude. The earlier measurement by Dall et al. (2010) is also shown with a yellow triangle.

\section{Possible dynamo explanation}

It is interesting to investigate the possibility that an $\alpha^{2}$ or $\alpha \Omega$ dynamo mechanism is at work in this star (Krause \& Raedler 1980). In the standard $\alpha \Omega$ dynamo (Parker 1955), the combined action of shear and mirror-antisymmetric turbulence regenerates the initial poloidal field and closes the dynamo loop. The field configurations are in general rather complex, and the solutions are oscillatory. Conversely, if the shear is not dominant, the rotational helicity of the turbulence alone amplifies the seed field up to the observed values. The resulting field is static in general and the geometry is rather simple, mostly dipolar. Only the turbulent diffusivity $\eta$ can be nonzero for a nonrotating isotropic turbulence. Both mechanism are believed to be at work in the Sun (Bonanno 2013; Guerrero et al. 2016), although the effect of meridional circulation (together with a low-eddy diffusivity) is essential for reproducing the observed butterfly diagram.

The astrophysical parameters obtained in the previous sections provide us with important information about the evolutionary state of the star, in particular, with the extension of the convective zone and the efficiency of the turbulence. To this purpose we used the Catania version of the GARSTEC code (Weiss \& Schlattl 2008; Bonanno et al. 2002a) to determine the interior structure of the star. In particular, we evolved from the ZAMS a $2 M_{\odot}$ model with an initial He abundance and metal fraction $Y_{i}=0.3$ and $Z / X=0.045$, respectively, up to a final age of $0.965 \mathrm{Gyr}$ in order to match the observed radius and luminosity. No heavy-element diffusion was included, and we chose a mixing-length parameter $\alpha_{\mathrm{MLT}}=1.65$, as obtained from solar calibration.

The relative importance of rotation and turbulence is described by the Coriolis number $\Omega^{*}=2 \Omega \tau$, where $\tau$ is the convective turnover time and $\Omega$ is the surface angular velocity. In our case, this is on the order of unity in the bulk of the convection zone, and near the surface $\Omega^{*} \ll 1$, as expected for such a slow rotator. The antisymmetric component of the turbulent electromotive force can be approximated with a pseudo-scalar of the type $\alpha(r) \cos (\theta)$. In particular for a small Coriolis number (Ruediger \& Kichatinov 1993; Küker \& Rüdiger 1999),

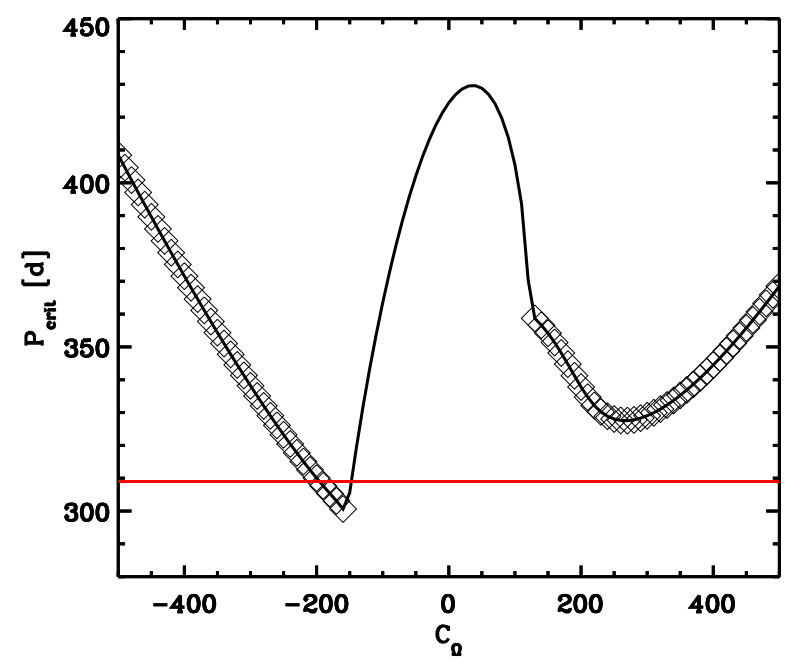

Fig. 7. Critical period as a function of $C_{\Omega}$, the critical dynamo number of the differential rotation. Negative $C_{\Omega}$ indicates that the angular velocity decreases outward. The diamond represents oscillatory solutions. The cycle periods are of about 50 years. The red line is the observed period.

$\alpha \approx \Omega^{*} \nabla \log (\rho) \tau u^{2} \approx \Omega \ell^{2} / R$ where $u$ is the (turbulent) convective velocity field and $\ell \approx 0.20 R$ is the characteristic radial scale of the turbulence.

The equipartition field $B_{\text {eq }}=\sqrt{4 \pi \rho} u$ is about $10^{3} \mathrm{G}$ at the bottom of the convection zone $\left(r_{\mathrm{b}}=0.4 R\right)$ and reaches $\sim 100 \mathrm{G}$ near the surface. Although this value is about the same as the observed value, it would be difficult to explain the generation of a large, long-lasting spot without a large-scale field that is produced by a suitable dynamo action. We can model our ignorance on the differential rotation by assuming a simple radial law of the type $\Omega=\Omega_{0}\left(r-r_{i}\right) / R$, where $\Omega_{0}$ is the observed equatorial angular velocity. With this definition, with a turbulent (eddy) diffusivity on the order of $\eta \sim 10^{14} \mathrm{~cm}^{2} \mathrm{~s}^{-1}$ (assuming the mixing-length relation $\eta=u \ell / 3$ ), the dynamo number of the shear $C_{\Omega}=\Omega_{0} R^{2} / \eta$ is about $250-300$.

Is the rotational period of EK Eridani short enough to support a dynamo action? A possible answer to this question can be obtained within the mean-field framework by solving the turbulent induction equation and determining the critical value of $\alpha$ that is required to obtain a self-sustained large-scale field. In particular, we used the CTDYN mean-field dynamo code presented in Bonanno et al. (2002b) that was further discussed and tested in Jouve et al. (2010). The results are depicted in Fig. 7. We computed the critical period $P_{\text {crit }}$ below which a dynamo action is possible, and this is plotted as a function of the rotational shear $C_{\Omega}$. Negative $C_{\Omega}$ implies that the angular velocity decreases in radius, diamonds represent critical oscillatory solutions, and the cycle periods range from 50 to $100 \mathrm{yr}$. A typical field configuration obtained with this approach is depicted in Fig. 8 for $C_{\Omega}=50$.

\section{Conclusions}

The star EK Eri is a unique laboratory for studying the behavior of acoustic pulsations in the presence of a large-scale magnetic field. This is clearly an important question that at the moment is far from being completely understood.

From the observational point of view, we confirm the $p$-mode detection that has been discussed in Dall et al. (2010), and we obtain a tentative measurement of the large separation for the 


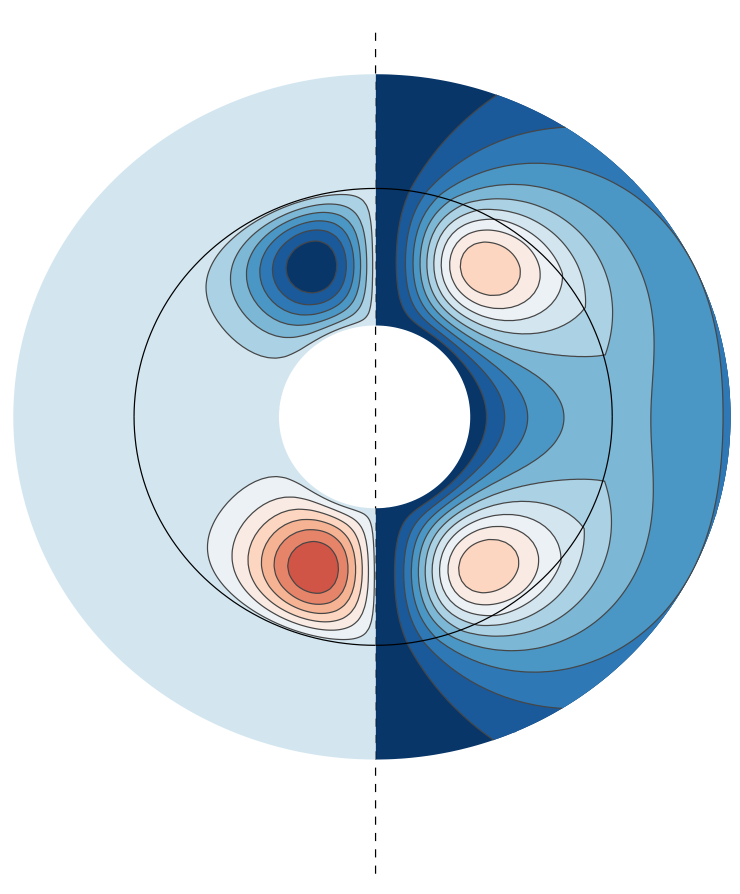

Fig. 8. Field topology for $C_{\Omega}=50$ (see text). The left hemisphere represents the isocontour lines of the toroidal field; blue levels show the negative and red levels the positive toroidal field. The right hemisphere represents the streamlines of the poloidal field. Blue levels show the counterclockwise and red levels the clockwise field lines. The black circle represents the stellar surface.

first time in this star. We also propose to modify the standard amplitude-luminosity variation of Kjeldsen \& Bedding (1995) in order to take the missing energy flux into account that is caused by a large spot.

Concerning the origin of the field in this object, we observe that the combined effect of rotation and turbulence is slightly supercritical and the dynamo action is thus possible. Moreover, a dynamo of the $\alpha^{2} \Omega$ type, or any dynamo characterized by an $\alpha$ tensor describing the standard mirror-antisymmetric turbulence, implies a dipolar-like magnetic field topology in the case of low values of $C_{\Omega}$. This is in agreement with observations, which suggest a dipolar topology of the field. Clearly, our result is heavily dependent on the assumed differential rotation. An approach based on numerical simulations is expected to clarify the question (Del Sordo et al., in prep.).

Acknowledgements. We would like to thank Tim Bedding for pointing out that the TESS data had become available for EK Eri while we were working on this paper. We thank the referee Thomas Kallinger for useful comments to our manuscript. E.C. is funded by the European Union's Horizon 2020 research and innovation program under the Marie Sklodowska-Curie grant agreement No. 664931. Based on observations made with the Hertzsprung SONG telescope operated on the Spanish Observatorio del Teide on the island of Tenerife by the Aarhus and Copenhagen Universities and by the Instituto de Astrofísica de Canarias. The work of F.D.S. has been performed under the Project HPCEUROPA3 (INFRAIA-2016-1-730897), with the support of the EC Research Innovation Action under the H2020 Programme; in particular, F.D.S. gratefully acknowledges the support and the hospitality of INAF Astrophysical Observatory of Catania.

\section{References}

Andersen, M. F., Grundahl, F., Christensen-Dalsgaard, J., et al. 2014, Revista Mexicana de Astronomia y Astrofisica Conference Series, 45, 83 Arentoft, T., Kjeldsen, H., Nuspl, J., et al. 1998, A\&A, 338, 909

Aurière, M., Konstantinova-Antova, R., Petit, P., et al. 2008, A\&A, 491, 499

Aurière, M., Konstantinova-Antova, R., Petit, P., et al. 2011, A\&A, 534, A139

Aurière, M., Konstantinova-Antova, R., Charbonnel, C., et al. 2015, A\&A, 574, A90

Bedding, T. R., Kjeldsen, H., Butler, R. P., et al. 2004, ApJ, 614, 380

Bonanno, A. 2013, Sol. Phys., 287, 185

Bonanno, A., Schlattl, H., \& Paternò, L. 2002a, A\&A, 390, 1115

Bonanno, A., Elstner, D., Rüdiger, G., \& Belvedere, G. 2002b, A\&A, 390, 673

Bonanno, A., Benatti, S., Claudi, R., et al. 2008, ApJ, 676, 1248

Bonanno, A., Corsaro, E., \& Karoff, C. 2014, A\&A, 571, A35

Brandenburg, A., \& Giampapa, M. S. 2018, ApJ, 855, L22

Brown, B. P., Miesch, M. S., Browning, M. K., Brun, A. S., \& Toomre, J. 2011, ApJ, 731, 69

Butler, R. P., Bedding, T. R., Kjeldsen, H., et al. 2004, ApJ, 600, L75

Chaplin, W. J., Bedding, T. R., Bonanno, A., et al. 2011, ApJ, 732, L5

Corsaro, E., \& De Ridder, J. 2014, A\&A, 571, A71

Corsaro, E., Grundahl, F., Leccia, S., et al. 2012, A\&A, 537, A9

Corsaro, E., Fröhlich, H.-E., Bonanno, A., et al. 2013, MNRAS, 430, 2313

Corsaro, E., De Ridder, J., \& García, R. A. 2015, A\&A, 579, A83

Corsaro, E., Mathur, S., García, R. A., et al. 2017, A\&A, 605, A3

Creevey, O., Grundahl, F., Thévenin, F., et al. 2019, A\&A, 625, A33

Dall, T. H., Bruntt, H., Stello, D., \& Strassmeier, K. G. 2010, A\&A, 514, A25

Dziembowski, W. A., \& Goode, P. R. 2005, ApJ, 625, 548

Elsworth, Y., Howe, R., Isaak, G. R., McLeod, C. P., \& New, R. 1990, Nature, 345,322

Fitzpatrick, E. L. 1999, PASP, 111, 63

Flower, P. J. 1996, ApJ, 469, 355

Frandsen, S., Jones, A., Kjeldsen, H., et al. 1995, A\&A, 301, 123

Fredslund Andersen, M., Pallé, P., Jessen-Hansen, J., et al. 2019, A\&A, 623, L9

García, R. A., Mathur, S., Salabert, D., et al. 2010, Science, 329, 1032

Gaulme, P., McKeever, J., Jackiewicz, J., et al. 2016, ApJ, 832, 121

Green, G. M., Schlafly, E. F., Finkbeiner, D. P., et al. 2015, ApJ, 810, 25

Grundahl, F., Fredslund Andersen, M., Christensen-Dalsgaard, J., et al. 2017, ApJ, 836, 142

Guerrero, G., Smolarkiewicz, P. K., de Gouveia Dal Pino, E. M., Kosovichev, A. G., \& Mansour, N. N. 2016, ApJ, 819, 104

Huber, D., Bedding, T. R., Arentoft, T., et al. 2011a, ApJ, 731, 94

Huber, D., Bedding, T. R., Stello, D., et al. 2011b, ApJ, 743, 143

Jacoutot, L., Kosovichev, A. G., Wray, A., \& Mansour, N. N. 2008, ApJ, 684, L51

Jouve, L., Brown, B. P., \& Brun, A. S. 2010, A\&A, 509, A32

Kallinger, T., De Ridder, J., Hekker, S., et al. 2014, A\&A, 570, A41

Kallinger, T., Beck, P. G., Stello, D., \& Garcia, R. A. 2018, A\&A, 616, A104

Kjeldsen, H., \& Bedding, T. R. 1995, A\&A, 293, 87

Kjeldsen, H., Bedding, T. R., Butler, R. P., et al. 2005, ApJ, 635, 1281

Krause, F., \& Raedler, K. H. 1980, Mean-field Magnetohydrodynamics and Dynamo Theory (Oxford: Pergamon Press)

Küker, M., \& Rüdiger, G. 1999, A\&A, 346, 922

Libbrecht, K. G., \& Woodard, M. F. 1990, Nature, 345, 779

Lindegren, L., Hernández, J., Bombrun, A., et al. 2018, A\&A, 616, A2

Palle, P. L., Regulo, C., \& Roca Cortes, T. 1989, A\&A, 224, 253

Parker, E. N. 1955, ApJ, 122, 293

Pires, S., Mathur, S., García, R. A., et al. 2015, A\&A, 574, A18

Ricker, G. R., Winn, J. N., \& Vanderspek, R. 2014, Society of Photo-optical Instrumentation Engineers (SPIE) Conference Series, 9143, 20

Ruediger, G., \& Kichatinov, L. L. 1993, A\&A, 269, 581

Sharma, S., Stello, D., Bland-Hawthorn, J., Huber, D., \& Bedding, T. R. 2016, ApJ, 822, 15

Stello, D., Chaplin, W. J., Basu, S., Elsworth, Y., \& Bedding, T. R. 2009, MNRAS, 400, L80

Stepien, K. 1993, ApJ, 416, 368

Strassmeier, K. G., Hall, D. S., Barksdale, W. S., Jusick, A. T., \& Henry, G. W. 1990, ApJ, 350, 367

Strassmeier, K. G., Stępień, K., Henry, G. W., \& Hall, D. S. 1999, A\&A, 343, 175

Weiss, A., \& Schlattl, H. 2008, Ap\&SS, 316, 99

Woodard, M. F., \& Noyes, R. W. 1985, Nature, 318, 449 